INPLASY

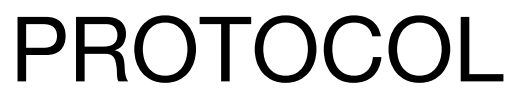

To cite: Sun et al. Music therapy for sleep quality in cancer patients with insomnia:

A protocol for systematic review and meta-analysis. Inplasy protocol 2021120128. doi:

10.37766/inplasy2021.12.0128

Received: 28 December 2021

Published: 28 December 2021

Corresponding author:

Yabin Gong

gongyabin@hotmail.com

Author Affiliation:

Yueyang Hospital of

Integrated Traditional Chinese

and Western Medicine,

Shanghai University of

Traditional Chinese Medicine.

Support: Shanghai 2021

Sailing Plan.

Review Stage at time of this submission: The review has not yet started.

Conflicts of interest:

None declared.

\section{Music therapy for sleep quality in cancer patients with insomnia: A protocol for systematic review and meta-analysis}

\author{
Sun, C1; Wang, Z2; Dai, Y3.
}

Review question / Objective: The aim of this systematic review is to compare music therapy in terms of efficacy in cancer patients with insomnia disorders to better inform clinical practice.

Condition being studied: The effectiveness of music therapy for cancer- associate insomnia is the main interest of this systematic review.

Information sources: MEDLINE (PubMed, Ovid) The Cochrane Library, Web of Science, Embase and Electronic retrieval of Chinese Biomedical Literature Database (CBM), China National Knowledge Infrastructure (CHKD-CNKI), VIP database, Wanfang Database will be searched from inception time to date. In addition, the included literature will be reviewed and relevant literature will be supplemented.

INPLASY registration number: This protocol was registered with the International Platform of Registered Systematic Review and Meta-Analysis Protocols (INPLASY) on 28 December 2021 and was last updated on 28 December 2021 (registration number INPLASY2021120128).

\section{INTRODUCTION}

Review question / Objective: The aim of this systematic review is to compare music therapy in terms of efficacy in cancer patients with insomnia disorders to better inform clinical practice.
Rationale: Cancer is the second leading cause of death in the world. Although the continuous progression of diagnosis and treatments brings hope to cancer patients, they are often accompanied by many mental symptoms during disease progression and treatments, such as 
fatigue, insomnia, anxiety, etc., which may affect the quality of life of patients. The overall incidence of insomnia in the common population was about $10 \%-20 \%$, with the total incidence of insomnia in cancer patients being $30 \%-60 \%$. Insomnia in cancer patients is a complex relationship between biological and psychological factors, which is associated with decreased quality of life, impaired immune function, and possibly disease progression. At present, people often do not pay attention to insomnia in cancer patients. Considering its adverse effects, it is particularly important to carry out safe and effective interventions with small side effects. For the treatment of cancer-related insomnia, cancer should be controlled first, followed by attention to the sleep status of patients. When patients experience insomnia, a variety of methods should be used for intervention. For cancer patients, the use of drugs needs to be more cautious. Therefore, exploring more effective non-drug therapies is now the direction of attention of clinicians. There have been many publications reporting the application of music therapy to sleep therapy in cancer patients, while there is a lack of evidence-based medicine. This study will systematically evaluate the efficacy of music therapy for cancer-related insomnia to provide an evidence-based basis.

Condition being studied: The effectiveness of music therapy for cancer- associate insomnia is the main interest of this systematic review.

\section{METHODS}

Search strategy: MEDLINE (PubMed, Ovid) The Cochrane Library, Web of Science, Embase and Electronic retrieval of Chinese Biomedical Literature Database (CBM), China National Knowledge Infrastructure (CNKI), VIP database, Wanfang Database. will be searched from inception time to date. In addition, the included literature will be reviewed and relevant literature will be supplemented. Only randomized controlled trials (RCTs) concerning the effects of music therapy for cancer patients with insomnia will be included in this metaanalysis. The search strategy will be established on the basis of MeSH/ Emtree and free-text terms. Details of the search strategy in PUBMED is as followed: Patients: ("cancer"[MeSH Terms] OR "carcinoma" [MeSH Terms] OR "cancer[Title/Abstract] OR "carcinoma[Title/Abstract]") AND ("insomnia"[MeSH Terms]) OR "agrypnia" [Title/Abstract] OR "aypnia"[Title/Abstract] OR "hyposomnia"[Title/Abstract] OR "sleeplessness"[Title/Abstract] OR "sleep disorder"[Title/Abstract] OR "sleep disturbance"[Title/Abstract] OR "lack of sleep"[Title/Abstract] OR "sleep deprivation"[Title/Abstract] OR "sleep deficiency"[Title/Abstract] OR "sleep insufficiency"[Title/Abstract] OR "insufficient sleep"[Title/Abstract]) OR "sleep"[Title/Abstract]). Intervention: "music therapy" [MeSH Terms] OR "music" [Title/Abstract] OR "melodiotherapy" [Title/ Abstract] OR "musical therapy" [Title/ Abstract] OR "Music care" [Title/Abstract] Studies: "clinical trial" [Publication Type] OR "clinical trials as topic" "[MeSH Terms] OR "clinical trial"[All Fields] OR "randomized controlled trial" [Publication Type] OR "randomized controlled trials as topic" [MeSH Terms] OR "randomized controlled trial"[All Fields] OR "randomised controlled trial"[All Fields]) OR "controlled clinical trial"[Publication Type] OR "controlled clinical trials as topic"[MeSH Terms] OR "controlled clinical trial"[All Fields] OR "multicenter study"[Publication Type] OR "multicenter studies as topic"[MeSH Terms] OR "multicenter study"[All Fields] OR "multicentre study"[All Fields]).

Participant or population: Patients enrolled in studies of cancer associated insomnia screening will be eligible for this review, with no exclusions based on ethnicity or age.Patients with pathological diagnosis of cancer; Patients diagnosed with cancer who meet the definition of insomnia by the American Academy of Sleep Disorders (ICSD-3) or Diagnostic and Statistical Manual of Mental Disorders, 5th edition, i.e., the enrolled population is all patients with cancer-related insomnia in the trial, 
regardless of the restriction of gender, age, race, condition duration, or degree of insomnia.

Intervention: The treatment group received music therapy, including various forms of music therapy, will be used in the treatment group.

Comparator: The control group received non-music therapy, including medication, yoga, placebo treatment, etc.

Study designs to be included: This study includes all randomized controlled trials of music therapy for cancer-associated insomnia patients.

Eligibility criteria: Any research whose full text is not available will be excluded.

Information sources: MEDLINE (PubMed, Ovid) The Cochrane Library, Web of Science, Embase and Electronic retrieval of Chinese Biomedical Literature Database (CBM), China National Knowledge Infrastructure (CHKD-CNKI), VIP database, Wanfang Database will be searched from inception time to date. In addition, the included literature will be reviewed and relevant literature will be supplemented.

Main outcome(s): We define in advance what types of outcomes are most relevant for the review question and how the outcomes will be measured, both in terms of the timing of measurement and type of scale. In this essay, sleep quality is defined as the main outcome. It will be measured by the Pittsburgh sleep quality index.

Data management: Literature data extraction for eligible studies that meet the inclusion criteria will be done by 2 authors independently and objections will be resolved by discussion with a third author. The following information will be extracted from the literature and recorded in a unified table: (1) basic information of the study: study name, first author, publication year, journal, and funded topic; (2) baseline characteristics of the study subjects (age, gender, sample size, body mass index, marital status, education, physical score, tumor disease, tumor stage, previous tumor treatment) and interventions (treatment time, course of treatment, frequency of treatment, mode of music therapy, mode of combined treatment, and follow-up time); (3) methodological information of the literature; (4) outcomes (evaluation methods of sleep quality, cancer patients' quality of life scores, pain scores, anxiety scores, and safety evaluation methods).

Quality assessment / Risk of bias analysis: Risk bias analysis will be assessed using the risk of bias assessment tool for RCTs in Cochrane SystemReview Manual5.1.0.

Strategy of data synthesis: To evaluate the progressive or diagnostic value of music therapy in lung cancer and compare nonmusic therapy between lung cancer patients and controls, we measured standardized mean difference (SMD) with $95 \%$ confidence interval $(95 \% \mathrm{Cl})$ as the effective value. A comprehensive systematic literature search in the Pubmed, and Embase was carried out the update to Dec.12, 2021. The standard mean difference (SMD), hazard ratio (HR), and $95 \%$ confidence interval $(95 \% \mathrm{Cl})$ were applied to investigate the effect sizes.

Subgroup analysis: If there is heterogeneity, subgroup analysis will be conducted according to the characteristics of patients included in the literature, such as gender, age, cancer type, stage, previous cancer treatment, etc.

Sensitivity analysis: In order to evaluate the stability of the results of meta-analysis, sensitivity analysis will be performed by deleting the included studies one by one to evaluate the change of the overall effect after deleting a certain study.

Language: Only randomized clinical trials published in English or Chinese will be considered for inclusion.

Country(ies) involved: China.

Keywords: music therapy, cancer, insomnia, protocol, meta-analysis. 
Dissemination plans: All data will be uploaded to the common data platform. When all data is finished, we will submit the research article.

Contributions of each author:

Author 1 - Chenbing Sun conceived the study and drafted the manuscript.

Email: greatbingbing@163.com

Author 2 - Zhe Wang contributed to the development of the selection criteria and statistical analyses.

Email: 2570377314@qq.com

Author 3 - Yuening Dai contributed to the search strategy and the risk of bias assessment strategy.

Email: 1733829039@qq.com 